CHRONIC OBSTRUCTIVE PULMONARY DISEASE

\title{
C-reactive protein in patients with COPD, control smokers and non-smokers
}

\author{
V M Pinto-Plata, H Müllerova, J F Toso, M Feudjo-Tepie, J B Soriano, R S Vessey, \\ B R Celli
}

See end of article for authors' affiliations

.....................

Correspondence to: Dr V M Pinto-Plata, Caritas St Elizabeth's Medical Center, 736 Cambridge St, Boston, MA 02135, USA; vpinto@copdnet.org

Received 8 February 2005 Accepted 16 August 2005 Published Online First 2 September 2005

\begin{abstract}
Background: Patients with chronic obstructive pulmonary disease (COPD) have raised serum levels of C reactive protein (CRP). This may be related directly to COPD and its associated systemic inflammation or secondary to other factors such as concomitant ischaemic heart disease (IHD) or smoking status. The aim of this study was to evaluate IHD and smoking as potential causes of raised CRP levels in COPD and to test the association between inhaled corticosteroid (ICS) use and serum CRP levels.

Methods: Cross sectional analyses comparing cohorts of 88 patients with COPD, 33 smokers (S), and 38 non-smoker (NS) controls were performed. Clinical assessments included a complete medical history, pulmonary function, 6 minute walk test $(6 \mathrm{MWT})$, cardiopulmonary exercise test, and high sensitivity serum CRP measurements.

Results: Serum CRP levels were significantly higher in patients with COPD $(5.03(1.51) \mathrm{mg} / \mathrm{l})$ than in controls (adjusted odds ratio 9.51 ; $95 \%$ confidence interval 2.97 to 30.45 ) but were similar in the two control groups (S: 2.02 (1.04) mg/l; NS: 2.24 (1.04) mg/l). There was no clinical or exercise evidence of unstable IHD in any of the subjects. CRP levels were lower in COPD patients treated with ICS than in those not treated $(3.7(3.0) \mathrm{mg} / \mathrm{l} \vee 6.3(3.6) \mathrm{mg} / \mathrm{l})$; this association was confirmed in an adjusted regression model $(p<0.05)$.

Conclusion: CRP levels are raised in COPD patients without clinically relevant IHD and independent of cigarette smoking, and reduced in patients with COPD using ICS. CRP may be a systemic marker of the inflammatory process that occurs in patients with COPD.
\end{abstract}

the increase in CRP is due to the presence of concomitant IHD or the systemic inflammatory processes associated with COPD. Limited information regarding other causes of increased CRP levels such as major infection, trauma, acute hospitalisation, lupus, inflammatory bowel disease, ${ }^{611} 12$ and the use of a low sensitivity assay to measure CRP were important limitations of the studies. ${ }^{11}{ }^{12}$ Indeed, none of the study protocols ruled out the presence of unstable angina or exercise induced cardiac ischaemia.

In a recent study $\operatorname{Sin} e t$ al $^{13}$ reported that the withdrawal of inhaled corticosteroids (ICS) resulted in a significant increase in CRP levels. However, no other information exists about the association between CRP level and the use of different types of ICS at different dosages.

Using a cohort observational design, we recruited patients with moderate to severe COPD and two control groups without COPD (smokers and non-smokers) and tested them using a high sensitivity CRP assay. We determined the presence of IHD and exercise induced ischaemia and excluded patients with a history of angina, infection, trauma, recent hospitalisation, connective tissue disorders, malignancy, and inflammatory bowel disease. The study design allowed us to determine the effect of smoking on CRP levels in subjects with and without airflow obstruction, as well as to assess the stability of CRP levels over time. We also evaluated the influence of ICS on the levels of CRP.

\footnotetext{
Abbreviations: COPD, chronic obstructive pulmonary disease; CRP, $\mathrm{C}$-reactive protein; $\mathrm{FEV}_{1}$, forced expiratory volume in 1 second; $\mathrm{FRC}$, functional residual capacity; FVC, forced vital capacity; ICS, inhaled corticosteroids; IHD, ischaemic heart disease; 6MWT, 6 minute walk test; TLC, total lung capacity; TLCO, carbon monoxide transfer factor; $\mathrm{VO}_{2}$ peak, peak oxygen consumption
} 


\section{METHODS}

\section{Study population}

This report is based on a cross sectional analysis of a cohort study at the Caritas St Elizabeth's Medical Center (CSEMC). Subjects were recruited between June 1999 and June 2001 from different sources (pulmonary clinic, pulmonary function test laboratory, local press advertisement, and referral by patients already enrolled). Control subjects consisted of responders to an advertisement in the local press and the hospital. They had no history of COPD, confirmed by spirometric tests performed during the screening process. The study was approved by the Institutional Review Board at CSEMC and all participants signed the informed consent.

The cohort consisted of three groups: (1) patients with COPD: smokers or ex-smokers with advanced disease (forced expiratory volume in 1 second $\left(\mathrm{FEV}_{1}\right)<55 \%$ predicted); (2) control smokers (S): current smokers with $\mathrm{FEV}_{1} / \mathrm{FVC}$ and $\mathrm{FEV}_{1}>70 \%$; and (3) control non smokers/ex-smokers (NS): patients with $\mathrm{FEV}_{1} / \mathrm{FVC}$ and $\mathrm{FEV}_{1}>70 \%$ who had either never smoked or had a smoking abstinence of more than 15 years and a smoking history of $<20$ pack years. The smoking status was confirmed by measuring the level of carboxyhaemoglobin in every subject. Inclusion criteria were age $>35$ years, stable disease for at least 2 months, and ability to complete the protocol. Exclusion criteria for patients and controls included myocardial infarction within the past 6 months, angina (Canadian class I-IV: chest pain with strenuous exercise, walking $>2$ blocks, walking $1-2$ blocks or at rest), congestive heart failure, ventilator dependency, malignancy, hepatic cirrhosis, end stage renal disease, rheumatoid arthritis, orthopaedic condition precluding performance of walking or cardiopulmonary exercise tests, neurological or psychiatric illness that interfered with participation in the study, tuberculosis, or any systemic infection or inflammatory process that could be associated with increased CRP values.

\section{Interview and physical examination}

One physician (VPP) interviewed all the patients using a designed questionnaire that includes direct questions on 40 co-morbid conditions, respiratory symptoms, current medications, anthropometric data, and a detailed physical examination. All subjects completed the St George's Respiratory Questionnaire. ${ }^{14}$

\section{Pulmonary function tests and dyspnoea}

Patients performed spirometric tests, lung volumes and carbon monoxide transfer favtor measurements following ATS standards. ${ }^{15}$ Functional dyspnoea was scored using the modified Medical Research Council scale. ${ }^{16}$

\section{Six minute walk test}

Patients performed the 6 minute walk test on two occasions following the ATS guidelines ${ }^{17}$ with some modifications (a person walked next to the patient and provided encouragement every 30 seconds). Oxygen saturation was monitored and supplemented to patients whose oxygen saturation decreased more than $<85 \%$ with the oxygen tank carried by the monitor. The longer of the two walks was used for the analysis.

\section{Cardiopulmonary exercise test}

Exercise testing was performed on a cycle ergometer while breathing room air (Vmax 29, Sensormedics; Yorba Linda, CA, USA). Patients completed the following protocol: 2 minutes rest, 3 minutes no-load work followed by a 16 Watts/min exercise with continuous 12 lead electrocardiogram (ECG) monitoring. Patients exercised until exhaustion or evidence of IHD was present (clinical or by ECG). Minute ventilation $(\mathrm{VE})$, oxygen uptake $\left(\mathrm{VO}_{2}\right)$, and carbon dioxide output $\left(\mathrm{VCO}_{2}\right)$ were measured breath by breath. The ECG was interpreted by an independent physician to evaluate the presence of exercise induced ischaemia. Patients with evidence of IHD were excluded and referred to the cardiology department.

\section{C-reactive protein}

Blood samples were obtained while the patients were at rest, before any other test was performed. Concentrations of CRP were determined using a high sensitivity chemiluminescent inmunoassay (DPC Immulite) following the manufacturer's instructions. The analytical sensitivity of this high sensitivity assay is of $0.1 \mathrm{mg} / \mathrm{l}$. Samples were identified by a bar code, so samples from controls and COPD patients were randomly distributed among assay plates.

\section{Statistical methods}

Normally distributed data were summarised using arithmetic means and standard deviations (SD). Variables judged to be skewed were log transformed and summarised using geometric means and associated standard deviations. Between-group differences in the main descriptive variables were analysed, depending on their nature, using the Student's $t$ test for independent samples or $\chi^{2}$ statistics (including Fisher's exact test).

We first tested the association between the level of CRP and disease status (COPD and non-COPD) and adjusted for potential covariates. Logistic regression model was fitted with the disease status as a response variable. Several variables were selected as covariates based on our preliminary knowledge that they can affect the CRP level: age, sex, smoking history (pack years), smoking status (never/former/current smoker), body mass index (BMI), and history of IHD. Odds ratios accompanied by the $95 \%$ Wald confidence intervals were recorded.

We then tested the reproducibility of CRP values over time. We selected a subgroup of subjects with longitudinal follow up (total $\mathrm{N}=78$; $\mathrm{COPD}=36, \mathrm{~S}=21, \mathrm{NS}=21$ ) and repeated the collection of serum samples (two samples each collected at respective visits; mean lag time between two visits 17.7 (7.5) months). Reproducibility was explored using the Bland-Altman plot expressing the change within a subject and mixed linear models (adjusted for age, sex, smoking history, BMI, $\mathrm{FEV}_{1}$, and ICS use). The zero hypothesis (no evidence for the lack of reproducibility) was tested as the absence of change over successive measurements. ${ }^{18}$

We next searched for the best predictors of CRP levels in COPD patients only using CRP levels as a response variable fitted in the generalised linear model. Predictor variables were selected based on our presumption that they are associated with COPD severity and patients' functions $\left(\mathrm{FEV}_{1}\right.$ predicted, TLCO predicted, 6-MWT, history of COPD exacerbations, MRC dyspnoea scale, and SGRQ). Known covariates (age, sex, smoking history (smoking status and pack years), history of IHD, and BMI) were also included. ${ }^{19} 20$ Predictors judged to show little contribution to the model $(p=0.15)$ were dropped from the full model.

Finally, we explored whether anti-inflammatory treatment with steroids can influence the CRP level in patients with COPD. The effect of medication, ICS, and oral steroids (binary variable) was tested using linear mixed models adjusted for age, sex, smoking status, $\mathrm{FEV}_{1}$ predicted, and BMI.

Between group differences were considered to be statistically significant at the 5\% level. The SAS Version 8.01 statistical software package was used for analyses. 


\begin{tabular}{|c|c|c|c|c|}
\hline & $\begin{array}{l}\text { COPD patients } \\
(\mathrm{n}=88)\end{array}$ & $\begin{array}{l}\text { Control } \\
\text { smokers (S) } \\
(\mathrm{n}=33)\end{array}$ & $\begin{array}{l}\text { Control } \\
\text { non-smokers (NS) } \\
(\mathbf{n}=38)\end{array}$ & $p$ value \\
\hline$M: F(\%)$ & $59: 41$ & $61: 39$ & $66: 34$ & \\
\hline Age (years) & 66 (9) & $62(6)$ & $67(7)$ & $\begin{array}{l}<0.05^{*} \\
<0.001 \dagger\end{array}$ \\
\hline BMI $\left(\mathrm{kg} / \mathrm{m}^{2}\right)$ & $27.04(5.43)$ & $26.54(5.63)$ & $28.94(4.43)$ & \\
\hline Smoking (packs/year) & $53(3)$ & $44(2)$ & $5(6)$ & $\begin{array}{l}<0.05^{*} \\
<0.001 \dagger \ddagger\end{array}$ \\
\hline IHD (\%) & 12.5 & 3.0 & 10.5 & \\
\hline MI (\%) & 10.1 & 3.0 & 2.6 & \\
\hline \multicolumn{5}{|l|}{ CRP (mg/l) } \\
\hline All subjects & $5.0(1.5)$ & $2.0(1.0)$ & $2.2(1.0)$ & $<0.001^{*} \ddagger$ \\
\hline $\begin{array}{l}\text { Subjects with IHD } \\
\text { excluded }\end{array}$ & $5.0(1.5)$ & $2.0(1.1)$ & $2.3(1.1)$ & $<0.001^{*} \ddagger$ \\
\hline \multicolumn{5}{|c|}{$\begin{array}{l}\text { IHD, ischaemic heart disease; Ml, myocardial infarction. } \\
\text { Results are expressed as mean (SD). CRP values are expressed as geometric mean (SD). } \\
\text { p values not significant unless stated. } \\
{ }^{*} \text { COPD } v \text { control smokers (S). †Smokers (S) v non-smokers (NS). } \neq \text { COPD v non-smokers (NS). }\end{array}$} \\
\hline
\end{tabular}

\section{RESULTS}

The baseline characteristics of the 88 COPD patients, 33 control smokers, and 38 control non-smokers are shown in table 1.

Patients with COPD were older and had longer smoking histories than control smokers (S). COPD and NS groups had the same prevalence of IHD but it occurred less frequently in the $S$ group. There was no difference between the groups in the prevalence of other screened co-morbidities (data not shown). Most of the COPD group were treated with inhaled short acting $\beta$ agonists and anticholinergics $(75 \%$ and $70 \%$, respectively). ICS (60\%) and long acting $\beta_{2}$ agonists were also prescribed $(41 \%)$. Patients received theophylline $(25 \%)$ and oral corticosteroids (16\%) less frequently.

The CRP level was significantly higher in the COPD group (geometric mean (SD) $5.03(1.51) \mathrm{mg} / \mathrm{l})$ than in $\mathrm{S}(2.02$ (1.04) $\mathrm{mg} / \mathrm{l}$ ) and NS (2.24 (1.04) mg/l; table 1, fig l) and this difference persisted even after adjustment for age, sex, smoking, history of IHD, and BMI in a multivariate logistic regression model. The adjusted odds ratio (OR) for increased CRP levels (log scale) comparing the COPD group and control subjects was 9.51 (95\% confidence interval (CI) 2.97 to 30.45). No difference was observed between S and NS control groups. Likewise, among COPD patients, the mean CRP level in smokers (14\%) and ex-smokers $(83 \%)$ was not significantly different (geometric mean 5.1 (3.2) mg/l $v 4.3$ (3.3) $\mathrm{mg} / \mathrm{l}$ ). There was also no difference in the CRP level after patients with known IHD were excluded from the analysis (table 1).

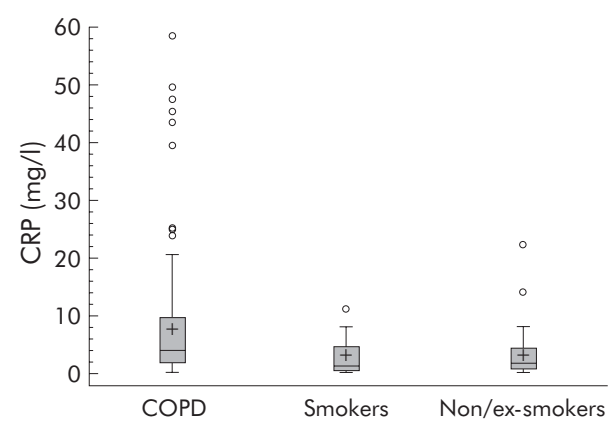

Figure 1 Geometric mean C-reactive protein (CRP) value distribution with interquartile range in COPD patients, control smokers and control non-smokers. CRP levels were higher in patients with COPD than in control smokers and non-smokers. The levels were similar in the two control groups.
The results of the pulmonary function tests, dyspnoea score, and exercise capacity assessments are summarised in table 2 .

The CRP levels remained essentially unchanged in a subgroup of patients ( 36 with COPD and 42 S+NS) retested at a subsequent visit (mean lag time between visits 17.7 (7.5) months). The coefficient of variance for variability within subjects was $14.6 \%$. Using the mixed linear regression model with adjustment for known covariates, no statistically significant difference was detected for the change in the CRP level over time (fig 2). The Bland-Altman plot with upper and lower limits (1.96 SD) also showed good consistency of data (difference between visit 1 and visit 2 ) with only six outliers (fig 3).

The correlation of CRP with potential predictors of severity and functioning of COPD and the ability of covariates to predict CRP levels was tested in the patients with COPD only. The final models describing predictive variables, their parameter estimate (regression coefficient) contribution to the sum of squares statistics, and significance are shown in table 3. This analysis showed that the 6MWT, age, and BMI significantly predicted the CRP level in the patients with COPD; the CRP level increased with increasing BMI and decreasing 6MWT and age. Sex, smoking history, FEV 1 \% predicted, and a history of COPD exacerbations in interaction with other predictors in the model contributed to predicting the CRP level, although none of them appeared to be an independent predictor with a $5 \%$ level of statistical significance. The most important clinically relevant predictor was 6MWT, which decreased with increasing CRP levels. Neither smoking status, MRC dyspnoea scale, TLCO, nor a history of IHD had an effect on CRP levels.

Fifty three patients with COPD (60\%) were using metered dose ICS. The baseline characteristics of both groups are shown in table 4 . The ICS used by the patients, dosage, and percentage of users included: fluticasone $440-880 \mu \mathrm{g}(45 \%)$; flunisolide 1000-4000 $\mu \mathrm{g}$ (29\%); budesonide 800-1200 $\mu \mathrm{g}$ (16\%); and triamcinolone: 400-1600 $\mu \mathrm{g}$ (10\%). The CRP level in ICS users was significantly lower than that of patients not using ICS when tested by Student's $t$ test (geometric mean 3.7 (3) $\mathrm{mg} / \mathrm{l} v 6.3$ (3.6) $\mathrm{mg} / \mathrm{l}, \mathrm{p}<0.05$; fig 4). The only statistically significant difference between ICS users and non-users was a higher pack year history in ICS non-users (table 4). Further analyses using a linear mixed regression model adjusted for age, sex, BMI, smoking history, FEV $_{1}$ predicted value, and oral steroid use showed a trend for a decrease in CRP level in ICS users compared with non-users $(\mathrm{p}<0.05$, data not shown $)$. 
Table 2 Results of the pulmonary function tests, dyspnoea level, and exercise capacity assessments of study population

\begin{tabular}{|c|c|c|c|}
\hline & COPD patients & Control smokers (S) & Control non-smokers (NS) \\
\hline $\mathrm{FEV}_{1}(\%)$ & 37 (11) & $91(15)$ & $92(17)$ \\
\hline $\mathrm{FEV}_{1} / \mathrm{FVC}(\%)$ & $42(11)$ & $73(6)$ & $75(6)$ \\
\hline $\operatorname{TLC}(\%)$ & $130(23)$ & $101(18)$ & $102(20)$ \\
\hline FRC (\%) & $174(38)$ & $99(25)$ & 105 (29) \\
\hline TLCO (\%) & $52(21)$ & $89(22)$ & $96(20)$ \\
\hline MRC & $2.2(0.9)$ & $0.5(0.7)$ & $0.2(0.4)$ \\
\hline 6MWT (m) & $375(99)$ & $546(75)$ & $558(102)$ \\
\hline Watts & $64(29)^{\circ}$ & $119(37)$ & $131(41)$ \\
\hline $\mathrm{VO}_{2}$ peak $(\mathrm{ml} / \mathrm{kg} / \mathrm{min})$ & $10(4)$ & $20(5)$ & $21(7)$ \\
\hline \multicolumn{4}{|c|}{$\begin{array}{l}\text { FEV } 1 \text {, forced expiratory volume in } 1 \text { second; FVC, forced vital capacity; TLC, total lung capacity; FRC, functiono } \\
\text { residual capacity; TLCO, carbon monoxide transfer factor, MRC, Medical Research Council dyspnoea scale; } \\
6 \mathrm{MWT}, 6 \text { minute walk test; } \mathrm{VO}_{2} \text { peak, peak oxygen consumption. } \\
\text { Results are expressed as mean (SD); geometric means are presented for non-normally distributed variables. } \\
\text { All variables shown in table } 2 \text { were significantly different for COPD patients } v \text { controls. There was no difference } \\
\text { between the two control groups. }\end{array}$} \\
\hline
\end{tabular}

\section{DISCUSSION}

In this study we confirm that CRP levels are raised in patients with moderate to severe COPD. This increase seems to be independent of clinically significant IHD. It was also independent of cigarette smoking and reduced in patients using ICS, probably reflecting the systemic inflammatory process in patients with severe disease.

Several epidemiological studies have found a significant relationship between pulmonary function and cause specific mortality due to IHD. ${ }^{9-12}$ Sin and Man, ${ }^{11}$ using population based data from NHANES III, studied 6629 patients including 2070 with mild, moderate, and severe COPD. Using a low sensitivity CRP assay (lowest detectable level $0.22 \mathrm{mg} / \mathrm{dl}$ ) and a coding scheme to calculate a cardiac infarction injury score with a resting ECG, they concluded that moderate and severe COPD was associated with a higher level of CRP and increased occurrence of ischaemic changes on the ECG. Similarly, Mannino and co-workers, ${ }^{12}$ also using data from NHANES III, described an association between the severity of
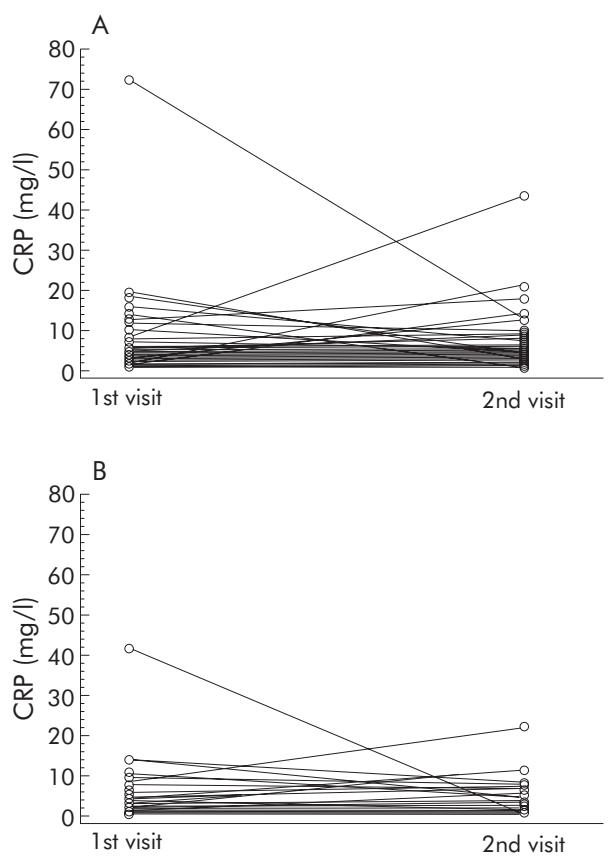

Figure 2 Intra-individual change in CRP level between visit 1 and visit 2 in (A) subjects with COPD and (B) controls with repeated measurement of CRP (mean lag time between visits 17.7 (7.5) months). Each line connects the measurements of an individual subject.
COPD and the level of CRP. A recognised limitation of both studies is that participants might have had undiagnosed or asymptomatic IHD that is associated with lower levels of pulmonary function and a high short term mortality risk. ${ }^{921}$

Our study expands these observations. Firstly, we excluded the presence of clinically significant or subclinical IHD using a comprehensive medical examination and exercise test with continuous ECG monitoring instead of a resting ECG alone. ${ }^{11}$ We also excluded other conditions associated with increased CRP levels including acute and chronic infection, chronic inflammatory diseases (rheumatological, gastrointestinal) and known malignancies. A follow up questionnaire and physical examination more than 1 year after the initial examination further excluded the possibility that any of these conditions were responsible for the increased level of CRP. Secondly, we used a high sensitivity CRP test to obtain more accurate values than in the two previous studies. ${ }^{11}{ }^{12}$

We observed no difference in the mean CRP level between control smokers and non-smokers, despite the fact that the former group was younger than the latter. Similarly, there was no difference among the patients with COPD who were current smokers and those who were ex-smokers. These findings may be due to a lack of power to determine a difference between the two groups, the absence of an inflammatory response in the smokers, or persistent inflammation in COPD patients even after smoking cessation as seen in human lung samples. ${ }^{22} \mathrm{~A}$ recent study ${ }^{23}$ did report an association between cigarette smoking and a high CRP concentration $(p<0.001)$, but the data were not shown. In

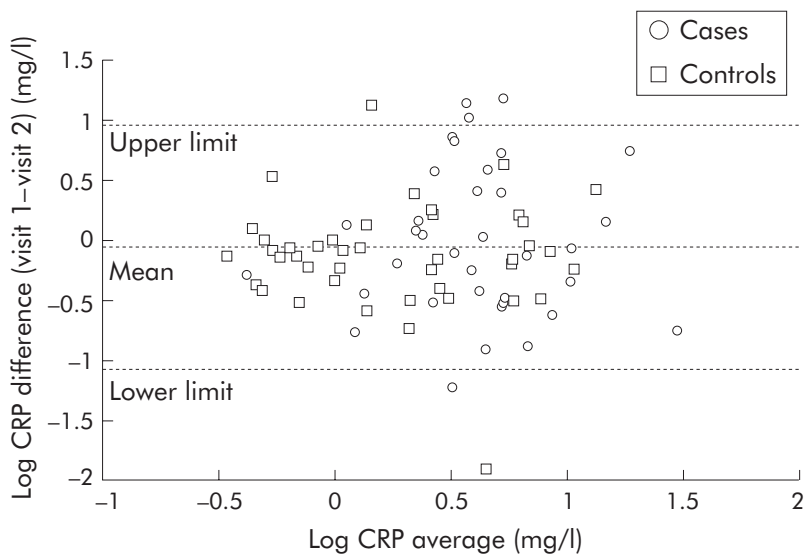

Figure 3 Bland-Altman plot of reproducibility of C-reactive protein (CRP) levels between two measurements. The CRP level remained stable over the mean 18 month follow up period. 
Table 3 Multivariate regression model evaluating the predictors of CRP levels in the COPD cohort

\begin{tabular}{lllll}
\hline & $\begin{array}{l}\text { Parameter } \\
\text { estimate }\end{array}$ & SE & Type III SS & Significance \\
\hline Age & -0.02 & 0.01 & 1.38 & 0.009 \\
Sex & -0.16 & 0.10 & 0.51 & 0.107 \\
Pack years & 0.18 & 0.12 & 0.43 & 0.141 \\
BMl & 0.04 & 0.01 & 2.84 & $<0.001$ \\
FEV 1 (\% predicted) & 0.81 & 0.43 & 0.67 & 0.065 \\
6MWT & -1.14 & 0.44 & 1.29 & 0.012 \\
COPD exacerbation history & -0.16 & 0.10 & 0.52 & 0.106 \\
\hline
\end{tabular}

$\mathrm{SE}$, standard error for the parameter estimate; SS, sum of squares; $\mathrm{BMI}$, body mass index; $\mathrm{FEV}_{1}$, forced expiratory volume in 1 second; $6 \mathrm{MWT}, 6$ minute walk test.

All of the effects were examined simultaneously (that is, the magnitude of each main effect or interaction was examined after controlling for all the other main effects and interactions).

The total sum of squares was $6.83 ; R^{2}$ estimate $=0.31$.

The following variables were included in the full model but excluded because they failed the significance limit $(p<0.15)$ : history of IHD, TLCO \% predicted, SGRQ composite score.

that study it was unclear if COPD was ruled out in the group of smokers who had a raised CRP level. The observation that CRP levels did not differ between smoker and non-smoker controls, and between smoker and ex-smoker patients with COPD, also suggests that the increase in CRP levels may represent a phenotype of CRP responders. Little is known about the reason why some persons exposed to cigarette smoke develop clinically important COPD whereas others do not. We speculate that there may be individuals with an "inherited" inflammatory genetic profile (a proportion of our control never smoker population had CRP levels above $3 \mathrm{mg} /$ l). If they are "exposed" to environmental factors (such as smoking or pollution), they have a higher risk of developing COPD.

Although our study fails to provide a mechanism, it does point out that the differences may precede the exposure to the trigger (cigarette smoking). It would be extremely interesting to determine CRP levels in teenagers before or around the time they begin to smoke to gain some insight into the presence in the general population of possible "inflammatory" phenotypes.

The CRP level in patients with COPD was lower in ICS users than in non-users (fig 4). These results are in agreement with a recent publication by $\operatorname{Sin} e t a l^{13}$ in which they showed that withdrawal of ICS from patients with mild and moderate disease was associated with a $71 \%$ increase in the baseline CRP level. It would be interesting to study larger groups of patients with COPD treated with $\mathrm{ICS}^{24}$ or withdrawn from $\mathrm{ICS}^{26}$ to determine if the changes in the number of COPD exacerbations and quality of life observed in those studies correlate with changes in the CRP level.

The mean CRP level in the patients with COPD in our study was similar to that reported by Mannino et al. ${ }^{12}$ It contrast,

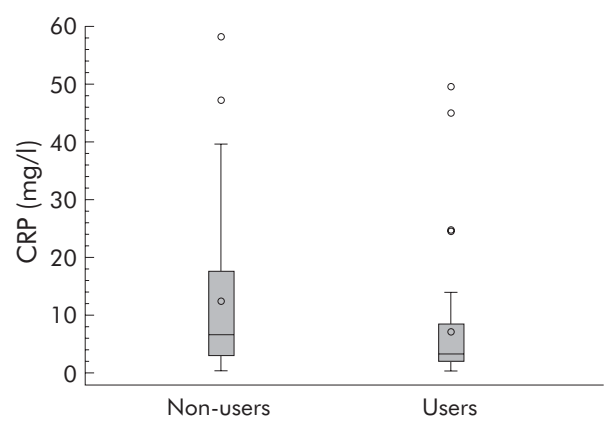

Figure 4 Geometric mean C-reactive protein (CRP) levels with interquartile range in users and non-users of inhaled corticosteroids (ICS). The CRP level was lower in users of ICS than in non-users. the values reported in epidemiological studies of controls and patients who eventually developed IHD is at least three times lower. ${ }^{23}$ This finding further supports the concept that the increase in the CRP level in patients with severe COPD cannot be attributed only to concomitant IHD. CRP is a marker of systemic inflammation and has been associated with an increased risk of incident myocardial infarction and stroke. ${ }^{68}$ As suggested by others, ${ }^{11}{ }^{13}$ it is possible that the persistent systemic inflammation in patients with COPD may contribute to the pathogenesis of atherosclerosis and cardiovascular disease and explain a high risk of cardiovascular mortality in patients with mild and moderate COPD. If we use the CRP levels of $<1,1-3$ and $>3 \mathrm{mg} / \mathrm{l}$ to represent low, moderate and high risk groups for future cardiovascular events, ${ }^{6}{ }^{19} 20$ almost $60 \%$ of the COPD patients in our study would be categorised as high risk (data not shown).

One important finding of our study is that the CRP level remained unchanged in the majority of patients retested after adjustment for age, sex, BMI, and $\mathrm{FEV}_{1} \%$ predicted. This finding is in agreement with a recent report ${ }^{23}$ that included 379 patients who provided paired blood samples for CRP analysis with a mean time between samples of 12 years and a within person correlation of 0.59 (95\% CI 0.52 to 0.66 ). Similar correlations have also been reported in other studies of normal subjects, ${ }^{27}$ and we have now reported it in patients with COPD.

There was no association between selected pulmonary function test measurements ( $\mathrm{FEV}_{1}$, TLCO $)$ and CRP levels in COPD patients. A probable explanation is that our study was limited to patients with moderate to severe disease in whom

Table 4 Demographic data and clinical characteristics of ICS users and non-users

\begin{tabular}{lll}
\hline & $\begin{array}{l}\text { ICS users } \\
(\mathbf{n}=53)\end{array}$ & $\begin{array}{l}\text { ICS non-users } \\
(\mathbf{n}=\mathbf{2 5})\end{array}$ \\
\hline Age (years) & $66(9)$ & $64(9)$ \\
Sex (\% men) & 60 & 56 \\
Smoking (packs/year) & $48(3)$ & $59(1)$ \\
Current smoker (\%) & 13 & 12 \\
IHD (\%) & 13 & 12 \\
$\mathrm{MI}(\%)$ & 11 & 8 \\
$\mathrm{BMI}\left(\mathrm{kg} / \mathrm{m}^{2}\right)$ & $26.7(5.2)$ & $27.58(6.4)$ \\
$\mathrm{FEV}(\%$ predicted) & $37(11)$ & $35(11)$ \\
$6 \mathrm{MWT}(\mathrm{m})$ & $388(96)$ & $345(111)$ \\
\hline
\end{tabular}

Results are expressed as mean (SD); geometric means are used for log transformed variables.

ICS, inhaled corticosteroids; IHD, ischaemic heart disease; MI, myocardial infarction; $\mathrm{BMI}$, body mass index; $\mathrm{FEV}_{1}$, forced expiratory volume in 1 second; $6 \mathrm{MWT}, 6$ minute walk test distance. 
the narrow range of these parameters probably failed to show any association. However, we found an association between the CRP level and the BMI and 6MWT that may prove clinically important. These clinical parameters probably reflect the systemic effects of COPD, and the CRP level is a measure of the systemic inflammation observed in patients with COPD.

Our study has some limitations. The patients with COPD included in the cohort were selected and therefore excluded patients in the community with milder forms of the disease. There were patients with known IHD in the COPD and control groups, but there was no difference in the mean CRP level when these patients were excluded. We carefully sought to exclude clinically important IHD, the biggest confounder of the studies published to date. The patients studied underwent a complete medical interview, a physical examination, and a cardiopulmonary exercise test with ECG monitoring to rule out possible unstable IHD and those with "silent" or subclinical IHD. However, the sensitivity and specificity of the exercise test in asymptomatic patients is unknown and may never be known because a coronary angiography, the "gold standard" for diagnosing coronary artery disease, is not indicated in asymptomatic patients. ${ }^{28}$ Patients with a recent acute exacerbation of COPD, arthritis, clinical evidence of acute or chronic infection, or other chronic inflammatory process were also excluded from the analysis. Our findings are in agreement with the larger epidemiological studies and serve to complement them. Finally, even though a large proportion of patients with COPD die from cardiac causes, ${ }^{13}$ many will die from COPD. ${ }^{5}$ To state simply that concomitant heart disease is the reason for the increased CRP levels in patients with COPD may not fully explain the findings reported here and may underestimate its importance as an independent biomarker of COPD.

In conclusion, we have confirmed that CRP levels are significantly raised in patients with COPD and this increase is not related to smoking per se or to concomitant clinical or subclinical IHD. The CRP level remains stable over time in normal individuals and in patients with COPD, and was lower in patients treated with ICS.

\section{Authors' affiliations}

V M Pinto-Plata, B R Celli, Pulmonary and Critical Care Division, Caritas St Elizabeth's Medical Center, Tufts University School of Medicine, Boston, MA, USA

H Müllerova, M Feudjo-Tepie, J B Soriano, GlaxoSmithKline R\&D, Worldwide Epidemiology Department, London, UK

J F Toso, R S Vessey, GlaxoSmithKline R\&D, Discovery Medicine Department, King of Prussia, PA, UK

This work was supported by a grant from GlaxoSmithKline.

Competing interests: none declared.

\section{REFERENCES}

1 Barnes PJ, Shapiro SD, Pauwels RA. Chronic obstructive pulmonary disease: molecular and cellular mechanisms. Eur Respir J 2003;22:672-88.

2 Barnes PJ. Medical progress: chronic obstructive pulmonary disease.

N Engl J Med 2000;343:269-80.
3 Vernooy JH, Kucukaycan M, Jacobs JA, et al. Local and systemic inflammation in patients with chronic obstructive pulmonary disease. Am J Respir Crit Care Med 2002; 166:1218-24.

4 Noguera A, Busquets X, Sauleda J, et al. Expression of adhesions molecules and $G$ proteins in circulating neutrophils in chronic obstructive pulmonary disease. Am J Respir Crit Care Med 1998;158:1664-8.

5 Celli BR, Cote CG, Marin JM, et al. The body-mass index, airflow obstruction, dyspnea and exercise capacity index in chronic obstructive pulmonary disease. N Engl J Med 2004;350:1005-12.

6 Ridker PM. Clinical application of C-reactive protein for cardiovascular disease detection and prevention. Circulation 2003;107:363-9.

7 Torres JL, Ridker PM. Clinical use of high sensitivity C-reactive protein for the prediction of adverse cardiovascular events. Curr Opin Cardiol 2003; 18:471-8.

8 Sesso HD, Buring JE, Rifai $N$, et al. C reactive protein and risk of developing hypertension. JAMA 2003;290:2945-51.

9 Schünemann HJ, Dorn J, Grant BJB, et al. Pulmonary function is a long term predictor of mortality in the general population. Chest 2000;1 18:656-64.

10 Hole DJ, Watt GCM, Davey-Smith G, et al. Impaired lung function and mortality risk in men and women: findings from the Renfrew and Paisley prospective population study. BMJ 1996;313:711-5.

11 Sin DD, Man SFP. Why are patients with chronic obstructive pulmonary disease at increased risk of cardiovascular diseases? Circulation 2003;107:1514-9.

12 Mannino DM, Ford ES, Redd SC. Obstructive and restrictive lung disease and markers of inflammation: data from the third National Health and Nutrition Examination. Am J Med 2003;1 14:758-62.

13 Sin DD, Lacy P, York E, et al. Effects of fluticasone on systematic markers of inflammation in chronic obstructive pulmonary disease. Am J Respir Crit Care Med 2004; 170:760-5.

14 Jones PW, Quirk FH, Baveystock CM. The St George's Respiratory Questionnaire. Respir Med 1991;85(Suppl B):25-31.

15 American Thoracic Society. Standardization of spirometry: 1994 update. Am J Respir Crit Care Med 1994;152:1107-36.

16 Bestall JC, Paul EA, Garrod R, et al. Usefulness of the Medical Research Council (MRC) dyspnoea scale as a measure of disability in patients with chronic obstructive pulmonary disease. Thorax 1999:54:581-6.

17 American Thoracic Society. ATS Statement: guidelines for the six-minute walk test. Am J Respir Crit Care Med 2002;166:111-7.

18 Bland JM, Altman DG. Measuring agreement in method comparison studies. Stat Methods Med Res 1999:8:135-60.

19 Pearson TA, Mensah GA, Alexander RW, et al. Markers of inflammation and cardiovascular disease: application to clinical and public health practice: a statement for healthcare professionals from the Centers for Disease Control and Prevention and the American Heart Association. Circulation 2003; 107:499-511.

20 Ridker PM, Rifai N, Rose L, et al. Comparison of C-reactive protein and lowdensity lipoprotein cholesterol levels in the prediction of first cardiovascular events. N Engl J Med 2002;347:1557-65.

21 Beaty TH, Cohen BH, Newill CA, et al. Impaired pulmonary function as a risk factor for mortality. Am J Epidemiol 1982;116:102-13.

22 Retamales I, Elliot WM, Meshi B, et al. Amplification of inflammation in emphysema and its association with latent adenoviral infection. Am J Respir Crit Care Med 2001;164:469-73.

23 Danesh J, Wheeler JG, Hirschfield GM, et al. C-reactive protein and other circulating markers of inflammation in the prediction of coronary heart disease. N Engl J Med 2004;350:1387-97.

24 Burge PS, Calverley PMA, Jones PW, et al. Randomized, double blind, placebo controlled study of fluticasone propionate in patients with moderate to severe chronic obstructive pulmonary disease: the ISOLDE trial. BMJ 2000;320: 1297-303.

25 Paggiaro PL, Dahle R, Bakran I, et al. Multicentre randomized placebocontrolled trial of inhaled fluticasone propionate in patients with chronic obstructive pulmonary disease. International COPD Study Group. Lancet 1998;351:773-80.

26 van der Valk P, Monninkhof E, van der Palen J, et al. Effect of discontinuation of inhaled corticosteroids in patients with chronic obstructive pulmonary disease: the COPE study. Am J Respir Crit Care Med 2002;166:1358-63.

27 Ockene IS, Matthews CE, Rifai N, et al. Variability and classification accuracy of serial high sensitivity $\mathrm{C}$-reactive protein measurements in healthy adults. Clin Chem 2001;47:444-50.

28 Gibbons RJ, Balady GJ, Bricker JT, et al. Committee to update the 1997 exercise testing guidelines. ACC/AHA 2002 guideline update for exercise testing: summary article, Circulation 2002;106:1883-92. 